\title{
The distribution of ionized gas in early-type galaxies. II. The velocity field of the ionized gas ${ }^{\star, \star \star}$
}

\author{
W.W. Zeilinger ${ }^{1}$, A. Pizzella ${ }^{2}$, P. Amico ${ }^{3}$, G. Bertin ${ }^{4}$, F. Bertola ${ }^{2}$, L.M. Buson ${ }^{5}$, I.J. Danziger ${ }^{3,6}$, \\ H. Dejonghe ${ }^{7}$, E.M. Sadler ${ }^{8}$, R.P. Saglia ${ }^{9}$ and P.T. de Zeeuw ${ }^{10}$ \\ 1 Institut für Astronomie, Universität Wien, Austria \\ 2 Dipartimento di Astronomia, Università di Padova, Italy \\ 3 European Southern Observatory, Garching bei München, Germany \\ 4 Scuola Normale Superiore, Pisa, Italy \\ 5 Osservatorio Astronomico, Padova, Italy \\ 6 Osservatorio Astronomico, Trieste, Italy \\ 7 Sterrenkundig Observatorium, Universiteit Gent, Belgium \\ 8 Astrophysics Department, School of Physics, Univ. of Sydney, Australia \\ 9 Universitäts-Sternwarte München, Germany \\ 10 Sterrewacht Leiden, The Netherlands
}

Received December 20, 1995; accepted March 1, 1996

\begin{abstract}
We report observations of the velocity field of the ionized gas in the nearby ellipticals NGC 1453, NGC 2974, NGC 3962, NGC 4636, NGC 6868 and NGC 7097. This is part of an ESO Key Programme intended to derive the mass distribution of elliptical galaxies and to investigate the possible presence of dark matter. All galaxy spectra are characterized by LINER-type emission. The derived gas kinematics confirm the picture of a regular gaseous disk structure with ordered rotation. The gas velocity dispersion profiles decline rapidly from a high central value in the range of $\simeq 150$ to $\simeq 250 \mathrm{~km} / \mathrm{s}$.
\end{abstract}

Key words: galaxies: elliptical and lenticular, $\mathrm{cD}$ - galaxies: ISM - galaxies: structure

\section{Introduction}

Ionized gas is now commonly found in elliptical galaxies (Roberts et al. 1991; Shields 1991). Moreover, in a fair number of ellipticals the emitting region is extended (up to $5-10 \mathrm{kpc}$ from the nucleus) and the gas is distributed in a regular manner suggestive of a disk (Bertola 1992 and references therein). The morphology of the line emitting region often appears to be decoupled from that of the light distribution. Kinematical decoupling between gas and stars is also observed. These decouplings suggest that the gaseous material is acquired from outside. Gaseous disks in elliptical galaxies can be used to test the gravitational field of the host galaxy and to constrain its intrinsic shape (Caldwell et al. 1986; de Zeeuw \& Franx 1989; Bertola et al. 1991).

Send offprint requests to: W.W. Zeilinger, Institut für Astronomie, Türkenschanzstraße 17, A-1180 Wien, Austria

${ }^{\star}$ Based on observations collected at the European Southern Observatory, La Silla (Chile)

${ }^{\star \star}$ Tables of Appendices A and B are only available in electronic form at the CDS via anonymous ftp 130.79.128.5
In this work (Paper II) we study the kinematical properties of the ionized gas for six galaxies selected (Table 1) from a larger sample previously investigated from the point of view of the structure of the line emitting region (Buson et al. 1993, Paper I). The observations are part of an ESO Key Programme intended to determine mass-to-light ratios of nearby elliptical galaxies (Amico et al. 1993). The selected galaxies are considered as suitable candidates to host a regular gaseous disk on basis of the morphology of the ionized gas region. In a subsequent paper (Pizzella et al. 1996, Paper III) a dynamical study will be carried out in order to derive the mass distribution of these galaxies, thus constraining the possible presence of dark matter in elliptical galaxies.

In Paper I the morphology of the emission-line regions has been analyzed in detail by means of narrow-band images in the light of $\mathrm{H} \alpha+[\mathrm{N} \mathrm{II}] \lambda \lambda 6548,6584 \AA$. The main conclusions of this study can be summarized as follows: $[a]$ In most of the $15 \mathrm{E}$ and $\mathrm{S} 0$ galaxies studied, the extended emission-line gas appears to lie in an - with respect to the line-of-sight - inclined, extended disk; $[b]$ The major axes of the stellar and gaseous components are frequently 
Table 1. Programme galaxies

\begin{tabular}{|c|c|c|c|c|c|c|c|c|c|}
\hline \multirow[t]{2}{*}{ Object } & \multicolumn{2}{|c|}{ morph. type } & \multirow[t]{2}{*}{$B_{\mathrm{T}}^{a}$} & \multirow{2}{*}{$\begin{array}{c}c z^{a} \\
{[\mathrm{~km} / \mathrm{s}]}\end{array}$} & \multirow{2}{*}{$\begin{array}{l}R_{\mathrm{e}}^{a} \\
{\left[{ }^{\prime \prime}\right]}\end{array}$} & \multirow{2}{*}{$\begin{array}{c}D^{b} \\
{[\mathrm{Mpc}]}\end{array}$} & \multirow{2}{*}{$\begin{array}{c}\mathrm{PA}_{\text {stars }}^{b} \\
{\left[^{\circ}\right]}\end{array}$} & \multirow{2}{*}{$\begin{array}{c}\mathrm{PA}_{\text {gas }}^{b} \\
{\left[^{\circ}\right]}\end{array}$} & \multirow{2}{*}{$\begin{array}{l}i^{b} \\
{\left[^{\circ}\right]}\end{array}$} \\
\hline & RSA & $\mathrm{RC} 3$ & & & & & & & \\
\hline NGC 1453 & E0 & $\mathrm{E} 2-3$ & 12.6 & 3933 & 25 & 71.2 & $28 \pm 1$ & $152 \pm 4$ & 45 \\
\hline NGC 2974 & $\mathrm{E} 4$ & $\mathrm{E} 4$ & 11.9 & 2006 & 24 & 38.8 & $39 \pm 2$ & $59 \pm 15$ & 59 \\
\hline NGC 3962 & $\mathrm{E} 1$ & $\mathrm{E} 1$ & 11.6 & 1818 & 35 & 36.2 & $8 \pm 4$ & $59 \pm 5$ & 54 \\
\hline NGC 4636 & $\mathrm{E} 0 / \mathrm{SO}_{1}(6)$ & E0-1 & 10.4 & 927 & 89 & 21.7 & $159 \pm 8$ & $108 \pm 11$ & 47 \\
\hline NGC 6868 & $\mathrm{E} 3 / \mathrm{S}_{2 / 3}(3)$ & E2 & 11.7 & 2858 & 34 & 48.8 & $80 \pm 8$ & $28 \pm 6$ & 54 \\
\hline NGC 7097 & $\mathrm{E} 4$ & E5 & 12.6 & 2539 & 18 & 45.3 & $19 \pm 3$ & $169 \pm 8$ & 57 \\
\hline
\end{tabular}

a from RC3

$b$ from Paper I misaligned; $[c]$ The mass of ionized gas in disks is typically $10^{4}-10^{5}$ solar masses, or 10-100 times higher than in "normal" early-type galaxies of similar optical luminosity; $[d]$ The ionized gas is probably closely related to the cold (atomic and molecular) component of the ISM, and may represent much less than $1 \%$ of the mass of this component. If so, the kinematics of the ionized gas are likely to be dominated by the underlying cold gas disk and the amount of ionized gas which is seen will depend on the number of ionizing photons available rather than the amount of gas present; [e] The radio continuum emission from early-type galaxies with ionized gas disks is typically ten times more powerful than that observed in normal ellipticals, suggesting that an active nucleus may provide an extra source of ionization.

\section{Observations and data reduction}

Long-slit spectra for the six programme galaxies were obtained at different position angles in order to map the velocity field of the ionized gas. The $\log$ of observations is given in Tables 2 and 3 . The slit positions are indicated for each programme galaxy in Fig. 1 using the $\mathrm{H} \alpha$ $+[\mathrm{NII}]$ images from Paper I. The observations were carried out with the ESO $3.6-\mathrm{m}$ and the ESO/MPI 2.2-m telescopes. At the 3.6-m telescope a Boller \& Chivens Cassegrain spectrograph equipped with a CCD camera and a $624 \times 1024$ pixel RCA CCD $\left(15 \times 15 \mu \mathrm{m}^{2}\right.$ pixel size) was used. The scale perpendicular to the dispersion was $0.55^{\prime \prime} /$ pixel. The slit width was $1.5^{\prime \prime}$. The wavelength range was $\lambda \lambda 5860-6750 \AA$ covering the $\mathrm{H} \alpha$ $(\lambda 6562.817 \AA)$ and $[\mathrm{NII}](\lambda 6548.1 \AA, \lambda 6583.4 \AA)$ emission lines for the subsequent analysis. The spectral resolution was on average $\sigma_{i} \simeq 30 \mathrm{~km} / \mathrm{s}$ as determined from the FWHM of the helium-argon lines of the comparison spectra and the night sky emission lines on the science spectra. The 2.2-m telescope was equipped with EFOSC2 at the Cassegrain focus using a $1024 \times 1024$ pixel Thomson CCD $\left(19 \times 19 \mu \mathrm{m}^{2}\right.$ pixel size) yielding a scale of $0.332^{\prime \prime} /$ pixel. The long-slit spectra were obtained with EFOSC2 grism \#9 in order to have a similar wavelength range as for the B\&C spectra $(\lambda \lambda 5850-6950 \AA)$ covering also the [SII] $(\lambda 6717 \AA, \lambda 6731.3 \AA)$ doublet which was missed in the $\mathrm{B} \& \mathrm{C}$ spectra because of the redshift. The slit width was $1.0^{\prime \prime}$. The spectral resolution for the EFOSC2 spectra was $\sigma_{i} \simeq 50 \mathrm{~km} / \mathrm{s}$ determined in the same way as for the B\&C spectra. The seeing values given in Tables 2 and 3 result either from estimates during the observations or from direct images taken with EFOSC2 in the course of observations. It must be noted, however, that by accumulating also the errors introduced by telescope tracking and auto-guiding, a FWHM of $\simeq 2^{\prime \prime}$ must be generally assumed as typical spatial resolution.

Each science spectrum was bracketed by two heliumargon calibration spectra in order to verify shifts along the dispersion due to instrument flexure. No significant shift was detected for the EFOSC2 spectra, while for the $\mathrm{B} \& \mathrm{C}$ data a typical shift of $\simeq 0.5$ pixel was measured for a 90 minutes exposure. The science spectra were calibrated with the ESO image processing package MIDAS using standard procedures for bias and dark current subtraction together with flat field correction. Cosmic ray events were removed both with a filtering algorithm and by visual inspection. The wavelength calibration was performed on each science frame for each spectral row independently using the respective averaged helium-argon spectra as reference and fitting a third order polynomial. The rms error of the dispersion curve was typically $<0.1$ pixel. The sky spectrum was derived for each science spectrum fitting for each wavelength bin a second order polynomial to the border regions of the detector free of galaxy light. In the cases where two spectra at the same position angle taken with the same instrument setup were available, they were aligned with respect to the galaxy centre as determined by a gaussian fitting of the light profile and subsequently added. 

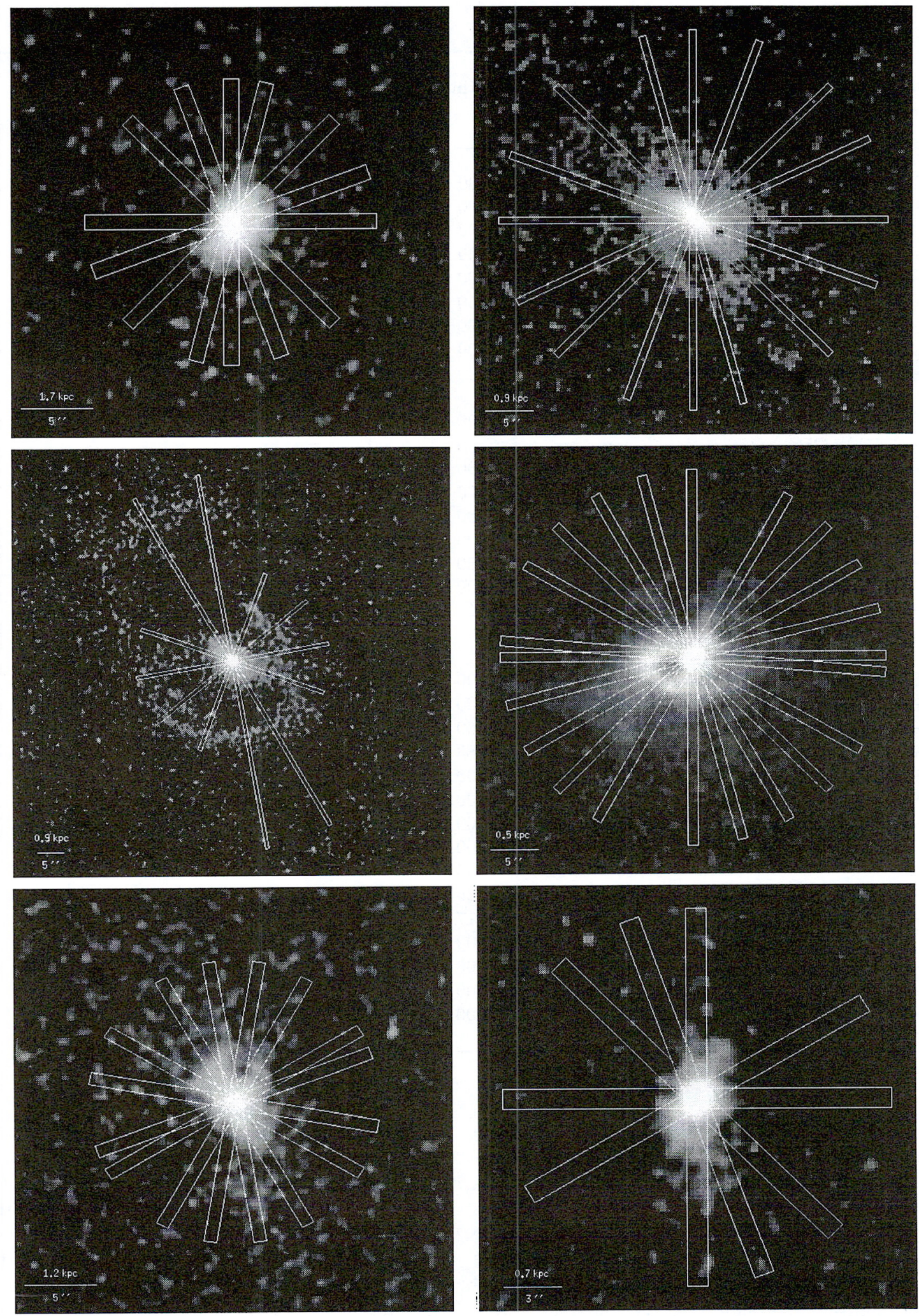

Fig. 1. Slit positions of the obtained spectra for NGC 1453, NGC 2974 (top row, left to right), NGC 3962 , NGC 4636 (centre row), NGC 6868 and NGC 7097 (bottom row) superimposed on the $\mathrm{H} \alpha+[\mathrm{N} \mathrm{II}]$ line emission images of Buson et al. 1993). A standard slit width of $1^{\prime \prime}$ is used as reference 
Table 2. Observing $\log -$ Part 1

\begin{tabular}{|c|c|c|c|c|c|c|}
\hline Object & $\begin{array}{l}\text { PA } \\
{\left[{ }^{\circ}\right]}\end{array}$ & telescope & date & $\begin{array}{l}\text { exp. time } \\
{\left[{ }^{\mathrm{s}}\right]}\end{array}$ & $\begin{array}{l}\text { seeing } \\
\text { FWHM }\end{array}$ & comments \\
\hline NGC 1453 & 0 & $3.6-\mathrm{m}$ & 13/14-Mar-91 & 5400 & $1.0^{\prime \prime}$ & \\
\hline$-11-$ & 20 & $2.2-\mathrm{m}$ & 6/7-Feb-92 & 5400 & $1.5^{\prime \prime}$ & \\
\hline$-11-$ & 45 & $3.6-\mathrm{m}$ & 12/13-Mar-91 & 3600 & $1.5^{\prime \prime}$ & \\
\hline$-1,-$ & 45 & $2.2-\mathrm{m}$ & 4/5-Feb-92 & 6600 & $1.0^{\prime \prime}$ & \\
\hline$-11-$ & 90 & $3.6-\mathrm{m}$ & 14/15-Mar-91 & 5400 & $1.5^{\prime \prime}$ & \\
\hline$-11-$ & 90 & $2.2-\mathrm{m}$ & 3/4-Feb-92 & 5550 & $1.1^{\prime \prime}$ & \\
\hline$-11-$ & 110 & $-11-$ & 7/8-Feb-92 & 5400 & $1.7^{\prime \prime}$ & \\
\hline$-n-$ & 135 & $3.6-\mathrm{m}$ & 15/16-Mar-91 & 5400 & $1.5^{\prime \prime}$ & \\
\hline$-11-$ & 165 & $2.2-\mathrm{m}$ & $5 / 6-F e b-92$ & 5400 & $1.0^{\prime \prime}$ & gas major axis $\left(152^{\circ}\right)$ \\
\hline NGC 2974 & 0 & $3.6-\mathrm{m}$ & 11/12-Mar-91 & 7200 & $1.0^{\prime \prime}$ & \\
\hline$-\infty-$ & 25 & $-11-$ & $-\prime-$ & 7200 & $1.2^{\prime \prime}$ & \\
\hline$-n-$ & 45 & $-\prime \prime-$ & 2/3-Mar-89 & 2400 & - & CCD rebinned to $1.65^{\prime \prime} /$ pixel \\
\hline$-n-$ & 70 & $-11-$ & 12/13-Mar-91 & 7200 & $1.5^{\prime \prime}$ & gas major axis $\left(59^{\circ}\right)$ \\
\hline$-\prime-$ & 90 & $-\prime \prime-$ & 11/12-Mar-91 & 7200 & $0.9^{\prime \prime}$ & \\
\hline$-11-$ & 90 & $-11-$ & 2/3-Mar-89 & 2400 & - & CCD rebinned to $1.65^{\prime \prime} /$ pixel \\
\hline$-\prime-$ & 115 & $-\prime \prime-$ & 12/13-Mar-91 & 7200 & $1.5^{\prime \prime}$ & \\
\hline$-n-$ & 135 & $-11-$ & 15/16-Mar-91 & 7200 & $1.5^{\prime \prime}$ & \\
\hline$-11-$ & 135 & $-1 \prime-$ & 2/3-Mar-89 & 6618 & - & CCD rebinned to $1.65^{\prime \prime} / \mathrm{pixel}$ \\
\hline$-11-$ & 160 & $-11-$ & 13/14-Mar-91 & 7200 & $1.5^{\prime \prime}$ & \\
\hline NGC 3962 & 10 & $3.6-\mathrm{m}$ & 15/16-Mar-91 & 7200 & $1.5^{\prime \prime}$ & \\
\hline$-n-$ & 10 & $-11-$ & 21/22-May-90 & 3600 & $1.0^{\prime \prime}$ & \\
\hline$-n-$ & 10 & $2.2-\mathrm{m}$ & $7 / 8-F e b-92$ & 5400 & $1.3^{\prime \prime}$ & clouds \\
\hline$-\prime-$ & 30 & $3.6-\mathrm{m}$ & 24/25-May-90 & 8883 & $2.5^{\prime \prime}$ & CCD rebinned to $1.65^{\prime \prime} /$ pixel, clouds \\
\hline$-11-$ & 30 & $2.2-\mathrm{m}$ & 4/5-Feb-92 & 5400 & $1.3^{\prime \prime}$ & \\
\hline$-\prime-$ & 30 & $-11-$ & $-\prime-$ & 3100 & $1.4^{\prime \prime}$ & stopped by sunrise \\
\hline$-n-$ & 40 & $3.6-\mathrm{m}$ & 22/23-May-90 & 7200 & $1.5^{\prime \prime}$ & gas major axis $\left(49^{\circ}\right)$ \\
\hline$-\prime \prime-$ & 40 & $-11-$ & 23/24-May-90 & 7200 & $1.0^{\prime \prime}$ & CCD rebinned to $1.65^{\prime \prime} /$ pixel \\
\hline$-11-$ & 70 & $-11-$ & 15/16-Mar-91 & 7200 & $1.5^{\prime \prime}$ & \\
\hline$-11-$ & 70 & $-11-$ & 21/22-May-90 & 5400 & $1.0^{\prime \prime}$ & \\
\hline$-\prime \prime-$ & 70 & $-11-$ & 24/25-May-90 & 6217 & $2.5^{\prime \prime}$ & CCD rebinned to $1.65^{\prime \prime} /$ pixel, clouds \\
\hline$-11-$ & 100 & $-11-$ & 22/23-May-90 & 7200 & $2.0^{\prime \prime}$ & \\
\hline$-11-$ & 130 & $-11-$ & 23/24-May-90 & 7200 & $1.5^{\prime \prime}$ & CCD rebinned to $1.65^{\prime \prime} /$ pixel, clouds \\
\hline$-\prime \prime-$ & 130 & $2.2-\mathrm{m}$ & 3/4-Feb-92 & 7200 & $1.3^{\prime \prime}$ & \\
\hline$-11-$ & 160 & $3.6-\mathrm{m}$ & 21/22-May-90 & 5400 & $1.0^{\prime \prime}$ & \\
\hline
\end{tabular}

In the subsequent analysis the [NII] $(\lambda 6583.4 \AA)$ line was used to derive the gas kinematics. An interactive procedure was used to define the continuum and fit the emission line with a gaussian for each spectral row. The measurements yielded line centers, FWHM of the fitted line and equivalent widths as a function of radius. From these measurements radial velocities and, subtracting the respective instrumental dispersion, velocity dispersions were derived. Several spectral rows were usually co-added in the outer parts of the galaxy in order to improve the $S / N$ of the measurements. In a few occasions, however, the recorded signal of the emission line was too faint to obtain a FWHM but the line center could be still reliably measured. The derived quantities, listed for each galaxy and each position angle, are given in Appendix A (Tables 1 to 10 for the radial velocity measurements) and in Appendix $\mathrm{B}$ (Tables 1 to 6 for the radial velocity dispersion measurements) as a function of distance from the galaxy center. The galaxy center was determined by fitting a gaussian to the galaxy light profile perpendicular to the dispersion. In order to smooth the velocity dispersion profile in the outer noisy parts, mean data points were calculated over intervals of usually a few arcseconds. The kinematic profiles are presented for each galaxy in Figs. 2-7. The systemic 
Table 3. Observing log - Part 2

\begin{tabular}{|c|c|c|c|c|c|c|}
\hline Object & $\begin{array}{l}\mathrm{PA} \\
{\left[^{\circ}\right]}\end{array}$ & telescope & date & $\begin{array}{c}\text { exp. time } \\
{\left[{ }^{\mathrm{s}}\right]}\end{array}$ & $\begin{array}{l}\text { seeing } \\
\text { FWHM }\end{array}$ & comments \\
\hline NGC 4636 & 0 & $3.6-\mathrm{m}$ & 14/15-Mar-91 & 7200 & $1.5^{\prime \prime}$ & \\
\hline$-\prime \prime-$ & 15 & $2.2-\mathrm{m}$ & $6 / 7-F e b-92$ & 3360 & $2.4^{\prime \prime}$ & clouds \\
\hline$-\prime \prime-$ & 30 & $3.6-\mathrm{m}$ & 13/14-Mar-91 & 7200 & $1.0^{\prime \prime}$ & \\
\hline$-\prime \prime-$ & 45 & $2.2-\mathrm{m}$ & $5 / 6-$ Feb-92 & 5400 & $1.1^{\prime \prime}$ & \\
\hline$-\mu-$ & 60 & $3.6-\mathrm{m}$ & 13/14-Mar-91 & 7200 & $1.0^{\prime \prime}$ & \\
\hline$-\prime \prime-$ & 85 & $2.2-\mathrm{m}$ & 5/6-Feb-92 & 5400 & $1.8^{\prime \prime}$ & \\
\hline$-\prime \prime-$ & 90 & $3.6-\mathrm{m}$ & 12/13-Mar-91 & 6000 & $1.5^{\prime \prime}$ & stopped by sunrise \\
\hline$-\prime \prime-$ & 90 & $2.2-\mathrm{m}$ & $6 / 7-F e b-92$ & 1800 & $1.4^{\prime \prime}$ & clouds \\
\hline$-\prime \prime-$ & 105 & $3.6-\mathrm{m}$ & 14/15-Mar-91 & 7200 & $1.5^{\prime \prime}$ & gas major axis $\left(108^{\circ}\right)$ \\
\hline$-\prime \prime-$ & 120 & $-n-$ & 11/12-Mar-91 & 7200 & $1.2^{\prime \prime}$ & \\
\hline$-\prime \prime-$ & 135 & $-n-$ & 14/15-Mar-91 & 4218 & $1.5^{\prime \prime}$ & stopped by sun rise \\
\hline$-\prime-$ & 135 & $2.2-\mathrm{m}$ & 7/8-Feb-92 & 5400 & $1.4^{\prime \prime}$ & \\
\hline$-11-$ & 150 & $3.6-\mathrm{m}$ & 12/13-Mar-91 & 7200 & $1.5^{\prime \prime}$ & \\
\hline NGC 6868 & 10 & $3.6-\mathrm{m}$ & 22/23-May-90 & 7200 & $1.5^{\prime \prime}$ & \\
\hline$-\prime-$ & 30 & $-\prime-$ & 21/22-May-90 & 7200 & $1.0^{\prime \prime}$ & gas major axis $\left(28^{\circ}\right)$ \\
\hline$-\prime \prime-$ & 30 & $-n-$ & 23/24-May-90 & 7200 & $2.0^{\prime \prime}$ & CCD rebinned to $1.65^{\prime \prime} /$ pixel \\
\hline$-\mu-$ & 60 & $-n-$ & 22/23-May-90 & 2700 & $1.5^{\prime \prime}$ & stopped by sun rise \\
\hline$-\prime \prime-$ & 80 & $-n-$ & 22/23-May-90 & 7200 & $1.5^{\prime \prime}$ & \\
\hline$-\mu-$ & 80 & $-n-$ & 24/25-May-90 & 3600 & $2.5^{\prime \prime}$ & CCD rebinned to $1.65^{\prime \prime} /$ pixel, clouds \\
\hline$-\prime-$ & 110 & $2.2-\mathrm{m}$ & 12/13-Jul-91 & 6503 & $1.0^{\prime \prime}$ & clouds \\
\hline$-\mu-$ & 120 & $3.6-\mathrm{m}$ & 21/22-May-90 & 6480 & $1.5^{\prime \prime}$ & stopped by sun rise \\
\hline$-\mu-$ & 150 & $2.2-\mathrm{m}$ & 14/15-Jul-91 & 5400 & $1.8^{\prime \prime}$ & cirrus clouds \\
\hline$-11-$ & 170 & $3.6-\mathrm{m}$ & 21/22-May-90 & 7200 & $1.0^{\prime \prime}$ & \\
\hline NGC 7097 & 0 & $2.2-\mathrm{m}$ & 13/14-Jul-91 & 5400 & $1.1^{\prime \prime}$ & clouds \\
\hline$-\prime-$ & 20 & $-n-$ & 14/15-Jul-91 & 5400 & $1.2^{\prime \prime}$ & \\
\hline$-\prime-$ & 45 & $-n-$ & 14/15-Jul-91 & 5400 & $1.4^{\prime \prime}$ & \\
\hline$-\prime \prime-$ & 90 & $-n-$ & 14/15-Jul-91 & 3600 & $1.7^{\prime \prime}$ & clouds \\
\hline$-11-$ & 110 & $-n-$ & 14/15-Jul-91 & 5400 & $1.4^{\prime \prime}$ & \\
\hline
\end{tabular}

velocity is subtracted from the radial velocities and the velocity dispersion is corrected for the instrumental dispersion.

Global estimates on the errors of the derived values were carried out by repeating the interactive measurements several times, testing in such a way the reliability of the obtained results for radial velocities and line widths (velocity dispersions). The line position measurements were found to be very robust, their major uncertainty being related to the $S / N$-ratio of the spectral line itself. For the high $S / N$ parts in the central regions of a galaxy a typical error of $\simeq \pm 5 \mathrm{~km} / \mathrm{s}$ was derived for radial velocities, in the outer parts the uncertainty increased towards $\leq \pm 15 \mathrm{~km} / \mathrm{s}$. The error estimates for the velocity dispersion measurements are found to be more complex. One of the major sources for uncertainties is the determination and subtraction of the continuum level in the spectrum and the shape of the respective emission line. In normal cases where the line profile could be well fitted by a single gaussian the errors were found to be $\leq \pm 10 \mathrm{~km} / \mathrm{s}$. An additional source of error is the accuracy of positioning the spectrograph slit on the center of the galaxy. It was assumed on basis of the narrow-band images that the peak of line emission coincides with the center in the continuum. As typical values one has to take into account inaccuracies on the level of $\simeq 1^{\prime \prime}$ for the $\mathrm{B} \& \mathrm{C}$ data because of a purely visual centering and $\simeq 0.5^{\prime \prime}$ for the EFOSC2 data where an imaging offset technique is used to position the slit.

The information on the radial intensity profile of the emission lines were used to constrain the viewing angle of the gaseous disk independently from the data in $\mathrm{Pa}$ per I under the assumption of a circular disk structure. In general a good agreement between the two data sets 


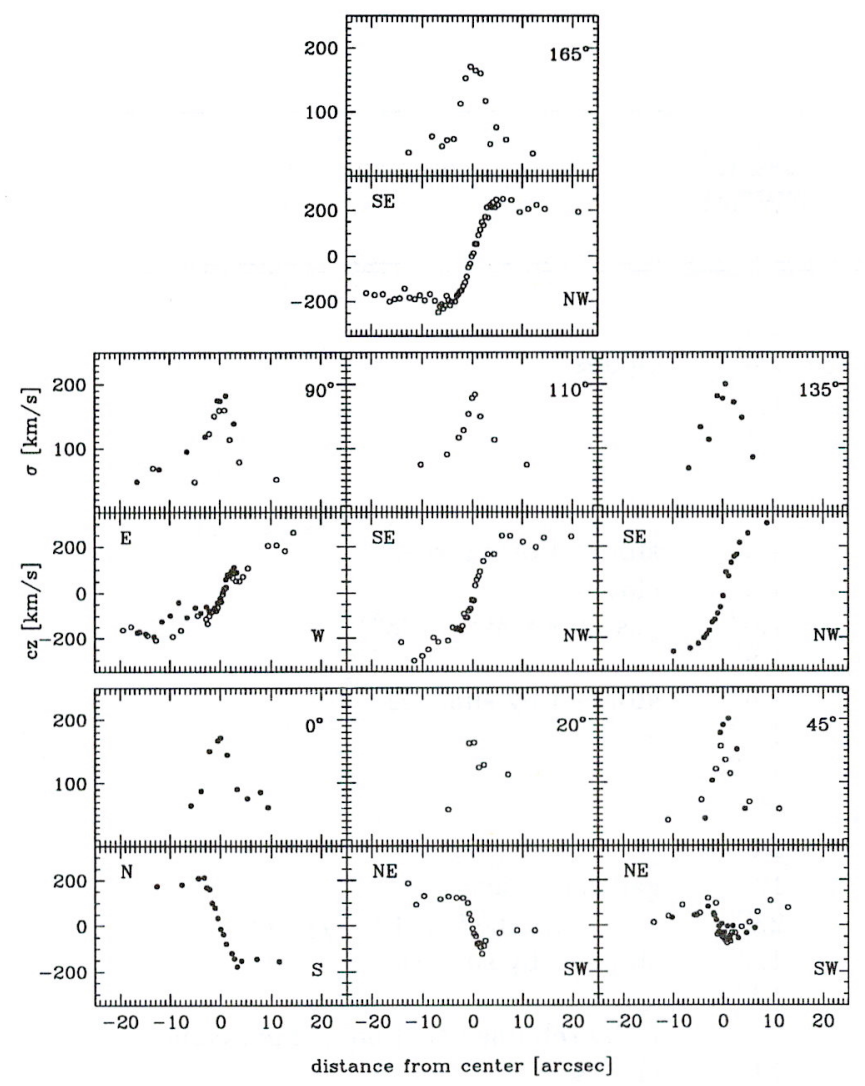

Fig. 2. Radial velocities and velocity dispersions as a function of distance from the galaxy center for NGC 1453 derived from the B\&C spectra (full points) and EFOSC2 spectra (open points)

were found taking into account the fact that the spectroscopic data are in general more radially extended then the narrow-band images.

\section{Results}

In this section, the results are presented and discussed for each programme galaxy. The spectra of all the galaxies are characterized by LINER-type emission indicating nuclear activity as described by Demoulin-Ulrich et al. (1984). The typical $[\mathrm{NII}] / \mathrm{H} \alpha$ ratios found are $\geq 2$. The kinematical data presented here give further decisive evidence in favour of the existence of regular gaseous disks with ordered rotation. The emission lines are generally characterized by large equivalent widths in the central parts. The derived velocity dispersion profiles generally fall off rapidly to almost the level of the instrumental resolution from a high central value. The typical peak values are in the range of $\simeq 150$ to $\simeq 250 \mathrm{~km} / \mathrm{s}$.

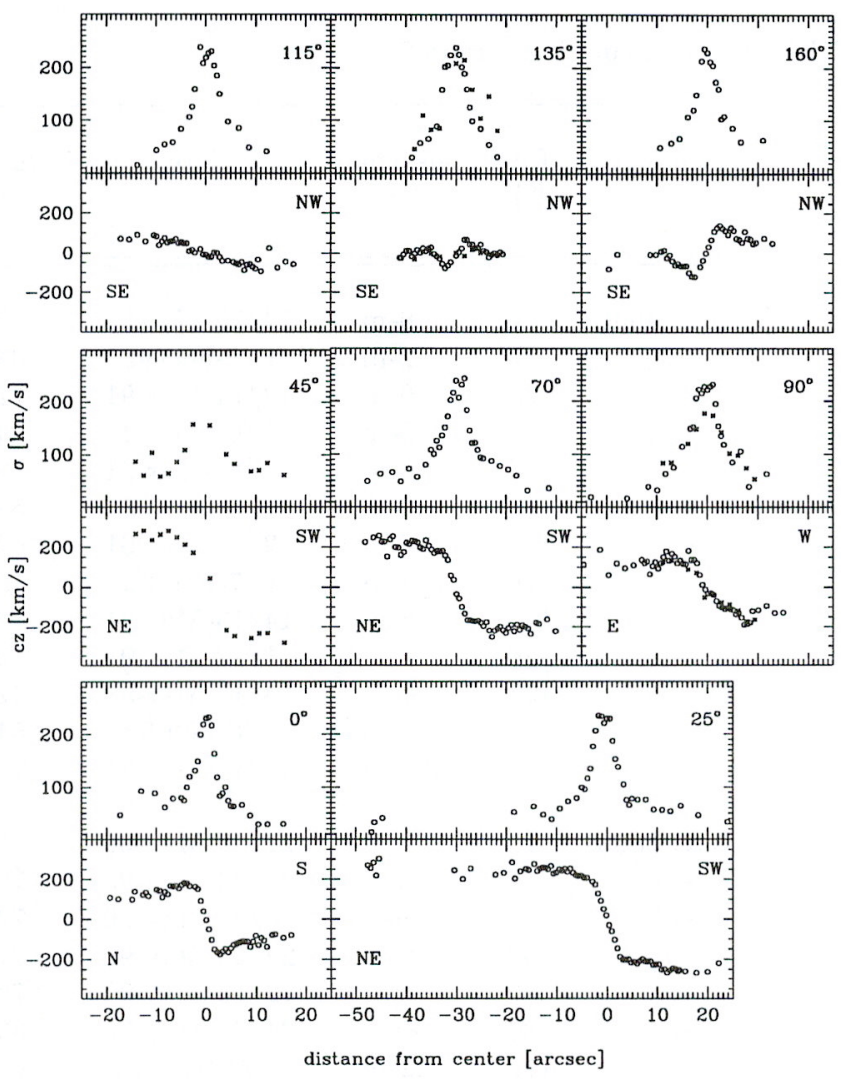

Fig. 3. Radial velocities and velocity dispersions as a function of distance from the galaxy center for NGC 2974 derived from the $\mathrm{B} \& \mathrm{C}$ spectra (open points). Crosses indicate $\mathrm{B} \& \mathrm{C}$ measurements with the detector rebinned in the spatial direction

\subsection{NGC 1453}

The analysis of the radial intensity profiles of the [NII] $\lambda 6583.4 \AA$ line indicates that the gaseous disk major axis lies at $\mathrm{PA} \simeq 131^{\circ}$ and that the inclination of the disk is $i \simeq$ $54^{\circ}$. The emission is detected in the spectra out to a radius of $20^{\prime \prime}$ from the nucleus and thus this analysis extends further out than the analysis of the $\mathrm{H} \alpha$ imaging described in Paper I. The velocity and the velocity dispersion fields of the gas are symmetric around the center of the galaxy indicating that the gas is moving in ordered orbits. The highest velocity is $V_{\max }=280 \mathrm{~km} / \mathrm{s}$ at $\mathrm{PA}=135^{\circ}$. The velocity dispersion profile of the gas has a central peak of about $200 \mathrm{~km} / \mathrm{s}$ and then falls rapidly off to a constant level of $\simeq 40 \mathrm{~km} / \mathrm{s}$ in the outer parts of the galaxy.

\subsection{NGC 2974}

The line emission is found to extend out to $30^{\prime \prime}$ from the galaxy nucleus. From the analysis of the radial intensity profiles of the [NII] $\lambda 6583.4 \AA$ line a position angle of $\simeq 48^{\circ}$ for the gaseous disk is derived and an inclination of $i=49^{\circ}$. The velocity and the velocity dispersion fields 
are symmetric around the center of the galaxy indicating that the gas is moving in ordered orbits. The presence of a significant velocity gradient $(\simeq 50 \mathrm{~km} / \mathrm{s} / \operatorname{arcsec})$ along the minor axis of the gaseous disk $\left(\mathrm{PA}=135^{\circ}\right)$ indicates as in the case of NGC 1453 a probably nonaxisymmetric galaxy potential. A maximum velocity of $V_{\max }=250 \mathrm{~km} / \mathrm{s}$ is measured at $\mathrm{PA}=25^{\circ}$. The velocity dispersion profile of the gas has a central peak of $\simeq 250 \mathrm{~km} / \mathrm{s}$ and then falls rapidly off to a constant level of $\simeq 70 \mathrm{~km} / \mathrm{s}$ in the outer parts of the galaxy. Because of the high velocity gradient the value of the central velocity dispersion may be overestimated due to seeing effects and due to the finite slit width.

NGC 2974 is the only galaxy of the sample which is also detected at $21 \mathrm{~cm}$. The orientation and kinematics of the neutral gas disk $\left(i=55^{\circ}, \mathrm{PA}=45^{\circ}, V_{\max }=355 \pm 60 \mathrm{~km} / \mathrm{s}\right.$ ( $=290 \mathrm{~km} / \mathrm{s}$ projected) (Kim et al. 1988) are in good agreement with the present data which confirm also previous kinematical studies of the ionized gas component (Demoulin-Ulrich et al. 1984; Kim 1989). NGC 2974 has an unresolved continuum source coincident with the galaxy center and is an X-ray source which has an extended halo (Forman et al. 1985).

In contrast to other ellipticals the gaseous disk is very well aligned with the stellar component and shares also similar kinematic properties. Perhaps not surprisingly, the galaxy also has a faint stellar disk (Scorza 1993; Cinzano \& van der Marel 1994). Evidence for spiral arm structures has been found in NGC 2974 (Bregman et al. 1992, Paper I).

\subsection{NGC 3962}

The morphology and kinematics of the ionized gas confirm the presence of two distinct subsystems revealed in Paper I. The inner gaseous disk shows regular kinematics with no evident peculiarities. The distinct emission knots originating from the arc-like structure are present in almost all position angles observed. The emission features of the arc-like structure are most evident in the position angles $10^{\circ}$ and $30^{\circ}$. A simple spherical analysis of the maximum velocities reached by the inner disk indicates that the disk major axis lies near $\mathrm{PA} \simeq 70^{\circ}$ and its inclination is $i \simeq 45^{\circ}$. Applying to the outer ring the same simple spherical analysis we find that the ring line of node is at $P A=21^{\circ}$. Even though the present data do not allow to deduce differences in the inclination $i$ between the ring and the disk, the misalignment of the line of nodes between the two structures is compelling. NGC 3962 is also known to have an unresolved radio source in the galaxy center (Birkinshaw \& Davies 1985).

\subsection{NGC 4636}

The ionized gas in NGC 4636 has a very complex structure which extends asymmetrically around the galaxy nu-

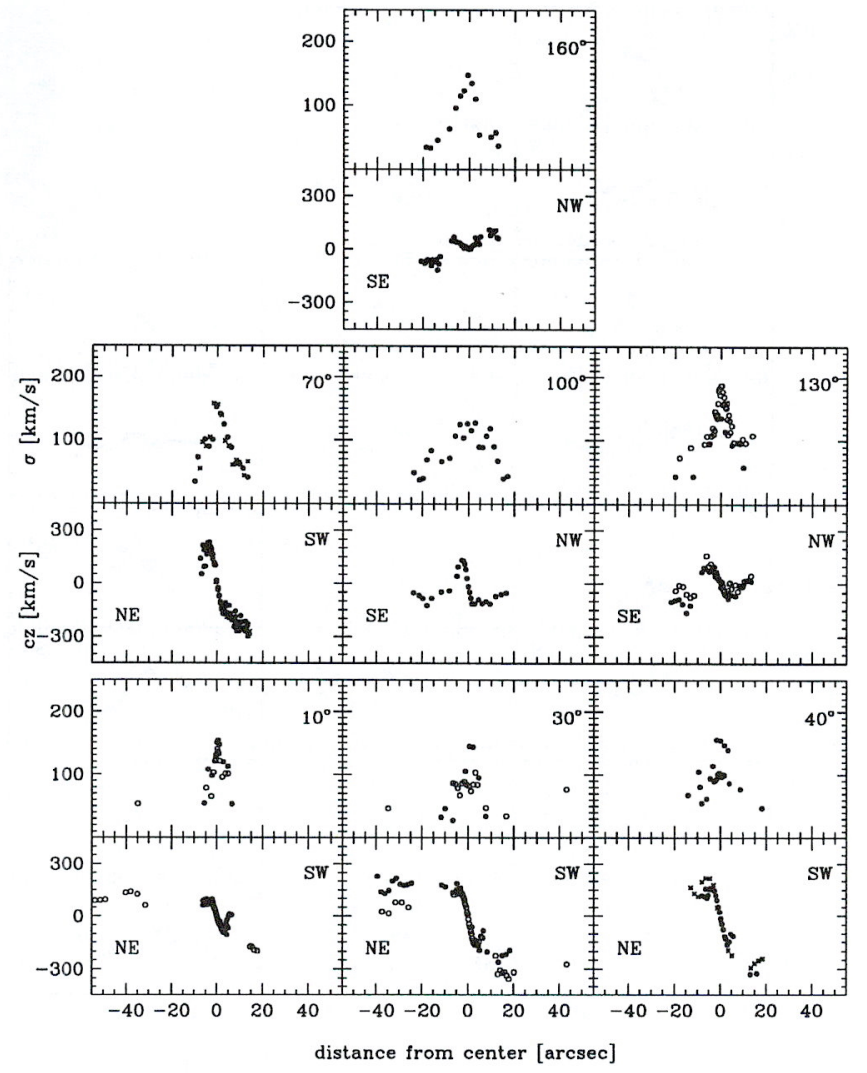

Fig. 4. Radial velocities and velocity dispersions as a function of distance from the galaxy center for NGC 3962 derived from the B\&C spectra (full points) and EFOSC2 spectra (open points). Crosses indicate $\mathrm{B} \& \mathrm{C}$ measurements with the detector rebinned in the spatial direction

cleus. Moreover, the $\mathrm{H} \alpha+[\mathrm{NII}]$ image indicates a "hole" of line emission coincident with the galaxy center. This peculiarity has been noted also by Demoulin-Ulrich et al. (1984). The complex morphology of the ionized gas is also reflected in the gas kinematics. The peak of the velocity dispersion $\left(\sigma_{\text {gas }} \simeq 220 \mathrm{~km} / \mathrm{s}\right)$ is about $3^{\prime \prime}$ offcenter from the galaxy nucleus. The velocity dispersion profile is rather asymmetric with a pronounced discontinuity at the galaxy center at $\sigma_{\text {gas }} \simeq 130 \mathrm{~km} / \mathrm{s}$. In the outer parts the gas kinematics of NGC 4636 tends to become rather chaotic. Also the rather high gas velocity dispersion in the outer parts indicates turbulent motions and/or material not settled.

The EFOSC2 data have been taken slightly $\left(\simeq 1.5^{\prime \prime}\right)$ offcenter missing the maximum of line emission and resulting in a flat velocity dispersion profile. The offset is probably due to not very favourable observing conditions (see Table 3). The apparent discrepancy of the two data sets at $\mathrm{PA}=90^{\circ}$ can be explained as due to different positionings of the spectrograph slit. Also the rotation is asymmetric with respect to the maximum of line emission. 

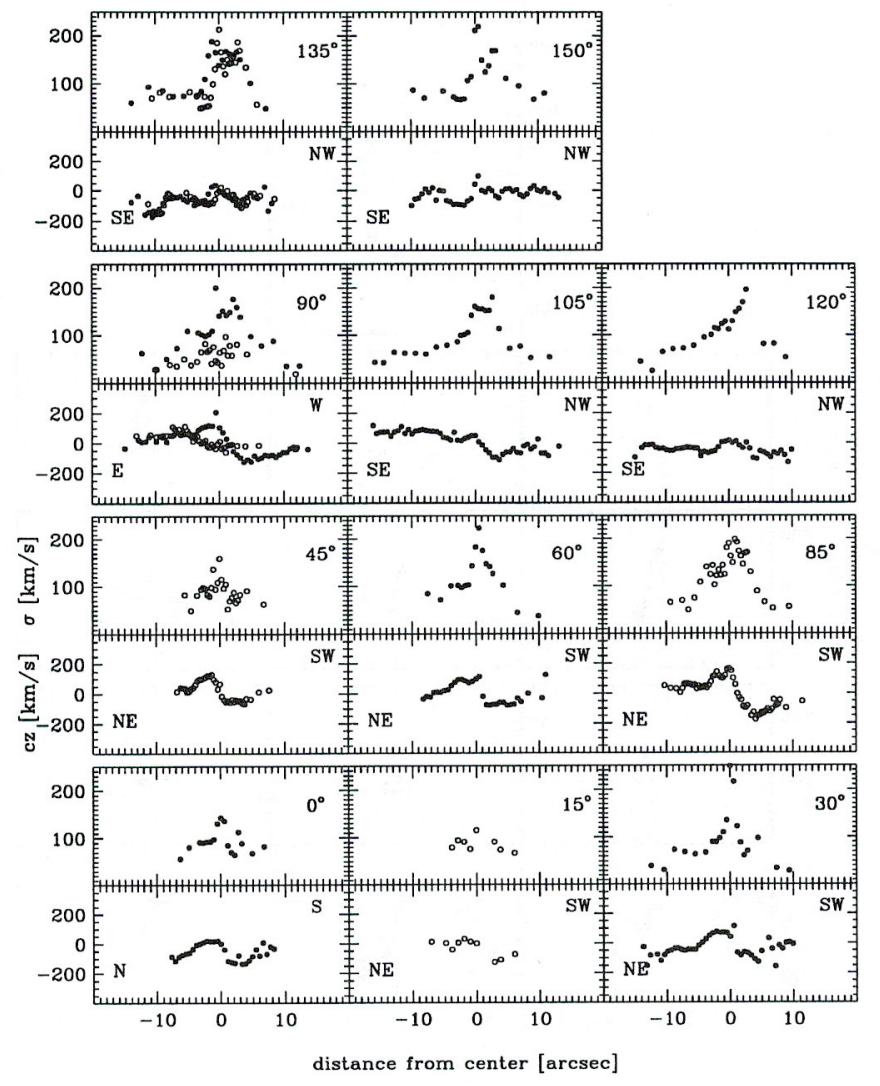

Fig. 5. Radial velocities and velocity dispersions as a function of distance from the galaxy center for NGC 4636 derived from the B\&C spectra (full points) and EFOSC2 spectra (open points)

$\mathrm{X}$-ray emission is detected in NGC 4636 extending well outside the optical galaxy (Trinchieri et al. 1994). NGC 4636 contains a jet-like radio source $\left(\mathrm{PA}_{\text {radio }}=33^{\circ} \pm\right.$ $8^{\circ}$ ) with a strong ridge of radio emission lying on either side of the nucleus. The ridge is resolved and shows a $\mathrm{S}-$ shaped bend about $5 \mathrm{kpc}$ from the nucleus (Birkinshaw \& Davies 1985). NGC 4636 is the strongest source in a field of 5 sources. Optically, the galaxy appears to be isolated.

\section{5. $N G C 6868$}

Even though the velocity fields and the velocity dispersion profiles of this galaxy are symmetric about the center, the gas is not moving in regular orbits. There is evidence in several position angles ( $\mathrm{PA}=80^{\circ}$ for instance) that the rotation curve does not remain flat in the outer parts but bends back towards a value close to the systemic velocity. At $\mathrm{PA}=110^{\circ}$ evidence for a distinct counter-rotating gas component is detected. The derived radial velocity curve (Fig. 6) has been obtained by fitting the line profile by means of a two gaussian component profile of instrumental widths. The unusual rotation curve near the gaseous disk minor axis at $\mathrm{PA}=120^{\circ}$ can be explained as the

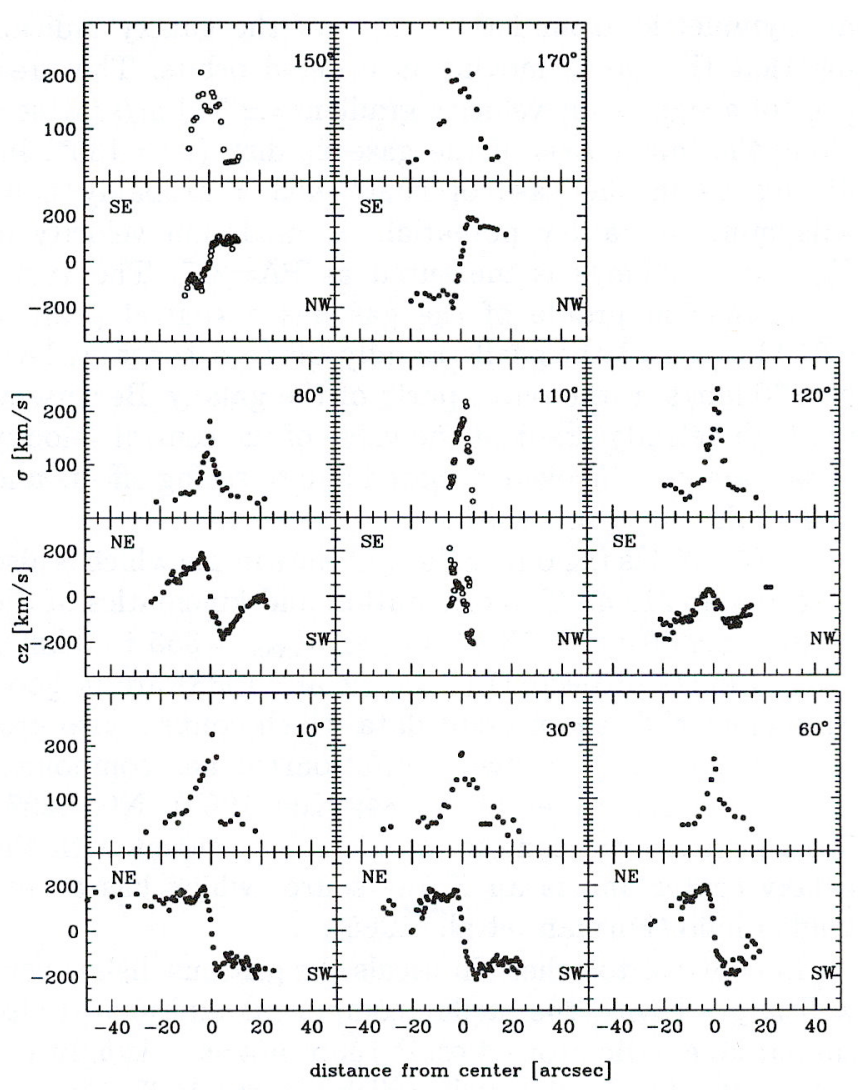

Fig. 6. Radial velocities and velocity dispersions as a function of distance from the galaxy center for NGC 6868 derived from the B\&C spectra (full points) and EFOSC2 spectra (open points)

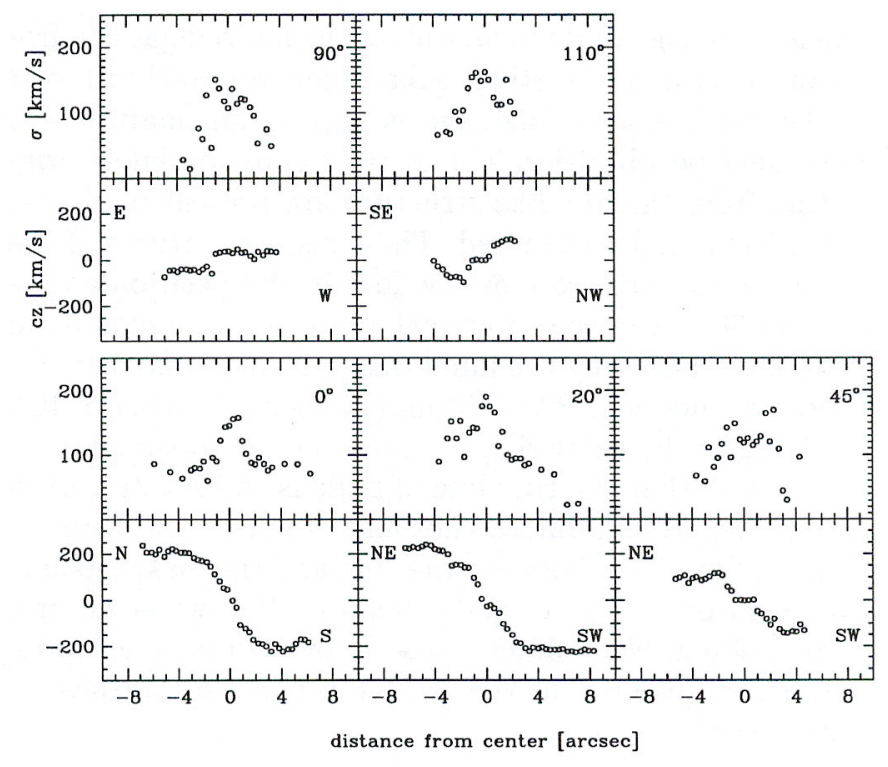

Fig. 7. Radial velocities and velocity dispersions as a function of distance from the galaxy center for NGC 7097 derived from the EFOSC2 spectra (open points) 


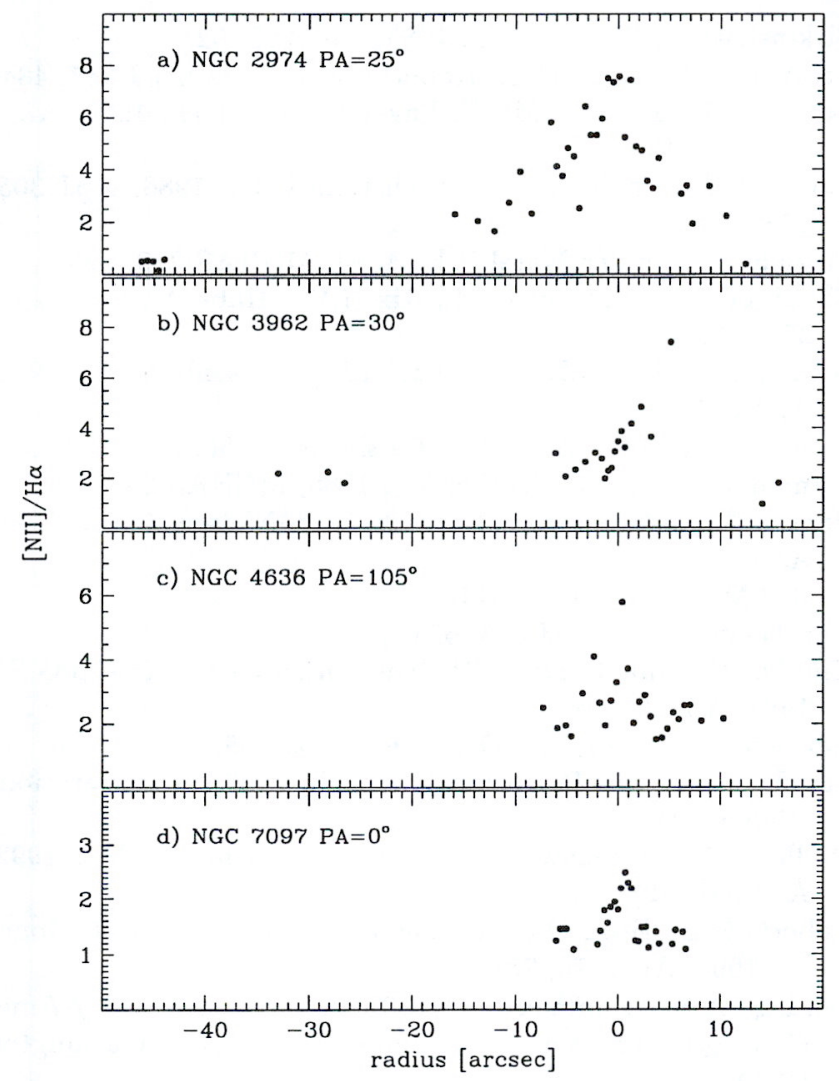

Fig. 8. Relative $[\mathrm{NII}] / \mathrm{H} \alpha$ flux ratios as a function of distance from the galaxy center for NGC 2974 a), NGC 3962 b), NGC 4636 c) and NGC 7097 d)

superposition of the curves of the two counter-rotating components, which are, however, not resolved. One component is dominating the central part of the curve and the other dominating the external part.

The velocity dispersion profile of the ionized gas is unusually narrow, peaked only in the region of the central velocity gradient, while in the outer parts of the gas rotation curve the line width of the emission lines is found to be essentially the instrumental profile.

\subsection{NGC 7097}

The analysis of the radial intensity profiles yields for the gaseous disk an inclination of $i=62^{\circ}$ with the line of nodes at $\mathrm{PA}=3^{\circ}$. The symmetry of the velocity curves about the center (only $\mathrm{PA}=110^{\circ}$ shows some asymmetry) makes this galaxy a good candidate for a complete dynamical study. Unfortunately only 5 different spectra are available for this galaxy. The spectra near the major axis $\left(\mathrm{PA}=0^{\circ}\right)$ extends up to $8^{\prime \prime}$ hardly reaching the turn-over of the velocity curve. Caldwell et al. (1986) interpreted their gas kinematical data as a centrally concentrated gaseous disk inclined $60^{\circ}$ to the plane of the sky in agreement with the present data. The gas rotation curve is found to have a steep central gradient with a pronounced discontinuity in the central part which may be related to the counterrotating stellar component.

\section{Discussion and conclusion}

The observed kinematics in the sample of six elliptical galaxies is consistent with the picture that (most of) the ionized gas is in a disk in regular rotation around the galaxy nucleus and follows closely the morphology of the line emitting region. The presence of large emission line widths in the central regions (interpreted as high velocity dispersions) however indicate, contrary to spiral galaxies, a rather hot gas component. A similar phenomenon has been already noted in S0 galaxies (Bertola et al. 1995). These observational facts lead to two obvious questions, namely the nature of the ionisation process and origin of the gaseous material. These two questions were also addressed in Paper I on basis of the results from the narrowband imaging. There it was noted that an unusually high fraction of the cold gas has been ionized. As possible additional ionizing source the active galaxy nucleus was identified, since the radio continuum emission was found to be typically $10-15$ times more powerful than normal elliptical galaxies of the same optical luminosity.

The relative flux ratios $[\mathrm{NII}] / \mathrm{H} \alpha$ were measured as a function of radius for those cases which show no significant absorbed $\mathrm{H} \alpha$ component, namely NGC 2974, NGC 3962, NGC 4636 and NGC 7097. The results are presented in Fig. 8.

The derived emission line ratios of the order [NII] $/ \mathrm{H} \alpha \geq 2$ exclude stellar radiation as the only source of ionization and suggest additional ionisation from a central non-thermal source in agreement with the conclusions of Paper I. Regarding the question of origin of the ionized gas component there are the following possibilities: either it could be material resulting from stellar mass loss which is not yet heated to the virial temperature of the galaxy (e.g. Bertola et al. 1984; Fillmore et al. 1986; Kent 1986; Kormendy \& Westpfahl 1989; Bertola et al. 1995), or material resulting from outside accretion, or a combination of both. Peculiar features such as the arc-like structure observed in NGC 3962, counter-rotating gas in NGC 6868 and peculiar features in the gas kinematics as in NGC 4636 are giving further support to the picture of an (ongoing) accretion event with the gaseous material settling into an equilibrium configuration. Hansen et al. (1991) note that the dust distribution of NGC 6868 closely follows that of Ly $\alpha$ emission-line gas detected with IUE and conclude that NGC 6868 has recently captured a gasrich galaxy. NGC 1453 is a more complex case, given the strong decoupling between gas and stars and no evidence of a zero velocity gradient at any observed position angle. The morphology of the gas, however, still indicates a very regular structure, suggesting material in an equilibrium 
configuration. This makes a non-axisymmetric (triaxial) galaxy potential likely.

NGC 2974 is a somewhat outstanding case from the observed sample: an emission region is detected at a distance of about $40^{\prime \prime}$ from the galaxy center at $\mathrm{PA}=25^{\circ}$ with $[\mathrm{NII}] / \mathrm{H} \alpha \simeq 0.5$ more typical of $\mathrm{H}$ II regions found in spiral galaxies. NGC 2974 appears to be the most "normal" case as the symmetry planes of gas and stars appear to be well aligned and no obvious decoupling is observed. This observational fact in combination with the reported spiral arm features seems to indicate a somewhat different nature with respect to the other galaxies of the sample. It must be also noted that NGC 2974 appears to be completely isolated in an $1^{\circ} \times 1^{\circ}$ field.

One of the central questions now is to what extent the gaseous disks observed in ellipticals are indeed settled and may be therefore used as a tracer of the potential of the underlying galaxy. These points will also be addressed in a forthcoming paper (Paper III).

Acknowledgements. AP acknowledges the support from an Acciaierie Beltrame grant. RPS acknowledges the financial support by the Deutsche Forschungsgemeinschaft under SFB 328 and 375 . WWZ acknowledges the support of the Austrian Fonds zur Förderung der wissenschaftlichen Forschung (project J0796-PHY).

\section{References}

Amico P., Bertin G., Bertola F., et al., 1993, in: Danziger I.J., Zeilinger W.W., Kjär K. (eds.), Structure, Dynamics and Chemical Evolution of Early-Type Galaxies. ESO, Garching, p. 225

Bertola F., 1992, in: Capaccioli M., Longo G., Busarello G. (eds.) Morphology and Physical Classification of Galaxies. Kluwer Academic Publishers Dordrecht, p. 115

Bertola F., Bettoni D., Rusconi L., Sedmak G., 1984, AJ 89, 356

Bertola F., Bettoni D., Danziger I.J., Sadler E.M., Sparke L.S., de Zeeuw P.T., 1991, ApJ 373, 369

Bertola F., Cinzano P., Corsini E.M., Rix H.-W., Zeilinger W.W., 1995, ApJ 448, L13
Birkinshaw M., Davies R.L., 1985, ApJ 291, 32

Bregman J.N., Hogg D.E., Roberts M.S., 1992, ApJ 387, 484

Buson L.M., Sadler E.M., Zeilinger W.W., et al., 1993, A\&A 280, 409 (Paper I)

Caldwell N., Kirshner R.P., Richstone D.O., 1986, ApJ 305, 136

Cinzano P., van der Marel R.P., 1994, MNRAS 270, 325

Christodoulou D.M., Katz N., Rix H.W., Habe A., 1992, ApJ 395,113

Demoulin-Ulrich M.H., Butcher H.R., Boksenberg A., 1984, ApJ 285, 527

Fillmore J.A., Boroson T.A., Dressler A., 1986, ApJ 302, 208

Forman W., Jones C., Tucker W., 1985, MNRAS 293, 102

Hansen L., Jørgensen H.E., Nørgaard-Nielsen H.U., 1991, A\&A 243, 49

Kent S.M., 1988, AJ 96, 514

Kim D.-W., 1989, ApJ 346, 653

Kim D.-W., Guhathakurta P., van Gorkom J.H., Knapp G.R., 1988, ApJ 330, 684

Kormendy J., Westpfahl D.J., 1989, ApJ 338, 752

Pizzella A., Amico P., Bertin G., et al., 1996 (in preparation) (Paper III)

Quillen A.C., de Zeeuw P.T., Phinney E.S., Phillips T.G., 1992, ApJ 391, 121

Roberts M.S., Hogg D.E., Bregman J.N., Forman W.R., Jones C., 1991, ApJS 75, 751

Sandage A.R., Tammann G., 1987, A Revised Shapley-Ames Catalog of Bright galaxies (2nd ed.), Carnegie, Washington (RSA)

Scorza C., 1993, in: Danziger I.J., Zeilinger W.W., Kjär K. (eds.) Structure, Dynamics and Chemical Evolution of Early-Type Galaxies. ESO, Garching, p. 115

Shields J.C., 1991, AJ 102, 1314

Steiman-Cameron T.Y., Kormendy J., Durisen R.H., 1992, AJ 104, 1339

Trinchieri G., Kim D.-W., Fabbiano G., Canizares C.R.C., 1994, ApJ 428, 555

de Vaucouleurs G., de Vaucouleurs A., Corwin H.G. Jr., et al., 1991, Third Reference Catalogue of Bright Galaxies. Springer Verlag, New York (RC3)

de Zeeuw P.T., Franx M., 1989, ApJ 343, 617 